\title{
Experiences of midwives and accoucheurs in implementation of pregnancy HIV guidelines in Limpopo province, South Africa
}

\author{
Maria S. Maputle ${ }^{1}$, Ireen T. Ramavhoya ${ }^{2}$, Lufuno Makhado ${ }^{1}$, Rachel T. Lebese ${ }^{1}$ \\ ${ }^{1}$ Department of Advance Nursing Science, University of Venda, Thohoyandou 0950, South Africa \\ ${ }^{2}$ Department of Nursing Science, University of Pretoria, South Africa
}

\begin{abstract}
Introduction: Each year, about 43,000 pregnant women and mothers die world-wide due to pregnancyrelated birth conditions. Most of these deaths are avoidable and preventable. An update on the maternal mortality of 2017 indicated that 976 pregnant women died between 2014 and 2016, despite being initiated on anti-retroviral drugs. Although, an improvement on human immunodeficiency virus (HIV) in pregnancy guidelines implementation was achieved, with $35 \%$ of maternal deaths occurring due to HIV infections, therefore there is a need to investigate the experience of midwives/accoucheurs regarding the implementation of HIV guidelines during pregnancy. The purpose of this study was to investigate and describe the experience of midwives/accoucheurs in implementing pregnancy HIV guidelines in the Limpopo province of South Africa.

Material and methods: This was a qualitative study using exploratory-descriptive design, and included professional nurses with midwifery qualification. Non-probability, purposive sampling was used for 18 participants based on data capacity. Data was collected through an in-depth, unstructured faceto-face interview and analyzed using Tesch's open coding method. Measures to ensure dependability, conformability, transferability, and credibility were applied.

Results: Three main subjects emerged from raw data, namely: experiences of midwives/accoucheurs during provision of care to HIV-positive pregnant women, management of pregnant HIV-positive women as per guidelines, and challenges experienced by midwives caring for pregnant HIV-positive women.

Conclusions: Midwives implemented the HIV guidelines during pregnancy; however, shortage of nurses during pregnant women's first visit resulted in prolonged waiting time for other patients. The prolonged time for patients' results and shortage of consulting rooms affected the implementation of HIV guidelines. The completion of different records was not accurately done. Non-adherence amongst clients affected the overall provision of HIV care.
\end{abstract}

HIV AIDS Rev 2020; 19, 2: 116-124

DOI: https://doi.org/10.5114/hivar.2020.96508

Key words: midwives/accoucheurs, implementation, HIV, pregnancy, experiences.

Address for correspondence: Prof. Maria S. Maputle, University of Venda, Private Bag X5050, Thohoyandou 0950,

South Africa, phone: +27159628125

e-mail: sonto.maputle@univen.ac.za
Article history:

Received: 21.04 .2019

Received in revised form: 16.07.2019

Accepted: 16.07.2019

Available online: 19.06 .2020
International Journal of HIV-Related Problems

HIV \& AIDS

R e vi e w 


\section{Introduction}

The implementation of human immunodeficiency virus (HIV) guidelines in pregnancy has increased from $52.6 \%$ to $98.8 \%$ as indicated by a study conducted at the Malamulo Hospital in Malawi on the prevention of mother to child transmission guidelines $[1,2]$. All pregnant women who tested positive for HIV were initiated on antiretroviral therapy (ART) [1]. However, with new infections, the rate of HIV guidelines implementation has decreased and has not yet reached the $90 \%$ target as required by the sustainable development goals of 2030. Each year, an average of 43,000 mothers die of birth complications despite the HIV/acquired immune deficiency syndrome (AIDS) knowledge provided to midwives $[3,4]$. According to the World Health Organization (WHO), maternal deaths of 287,000 per 100,000 livebirths were caused by pregnancy complications, including HIV infections [5]. In South Africa, the Saving Mothers report indicated an update of 800 pregnant women dying daily due to preventable causes though policies and guidelines provided to midwives/accoucheurs to follow during maternal health care services [6].

The report further revealed that two-third of deaths from non-pregnancy related-infection (NPRI) were due to HIV infections. Midwives indicated their competency in the implementation of HIV guidelines in pregnancy $[4,6]$. Of the pregnant women who died, $44.6 \%$ had a lowered cluster of differentiation 4 (CD4) count of less than 200 cells/ $\mathrm{mm}^{3}$ and in stage four, the opportunistic infections such as pneumonia and tuberculosis (TB) were the reasons for their death. Implementation of isoniazid (INH) initiation was low at $50 \%$ instead of $90 \%$ for HIV-positive women with negative TB results [7]. It was discovered that $79 \%$ were on ART drugs and $21 \%$ were not on ART. As such, sub-standard care, missed opportunities, and shortage of trained nurses were amongst the causes of an increase in maternal deaths due to HIV infections [8].

\section{Problem statement}

The rate of maternal mortality (MMR) in Limpopo compared to other provinces have declined steadily. The Saving Mothers report of 2017 showed a decline of $39.5 \%$ in 2014 to $26.3 \%$ in 2016 [8]. The Limpopo province was rated as number six, with $35 \%$ maternal deaths caused by HIV infections. Midwives/accoucheurs were implementing the HIV guidelines during pregnancy; however, the records assessed were often incomplete [7]. This was confirmed by the Saving Mothers report of 2014, which indicated that records of CD4 count and viral load results were missing in patients' files [7]. The report further indicated that pregnant women who were previously tested negative, tended to be positive at a later stage and as such, they were missed because their last date of HIV test was not documented on their files; therefore, no repeated tests were done [7]. The guidelines state that HIV testing of pregnant women must be done every 12 weeks until the cessation of breastfeeding of those who are acutely ill, regardless of their last day of the test. With the new developments, midwives are expected to be competent and to apply the latest HIV screening and treatment protocol in order to prevent HIV complications [8]. Irrespective of the efforts by the South African government on facilitating the implementation of HIV guidelines by midwives, their knowledge and skills need to be strengthened. Availability of updates on the management of HIV-positive pregnant women could contribute to the MMR declining. It was against this background that this study was conducted in order to investigate the experiences of midwives/ accoucheurs in implementing HIV guidelines during pregnancy in the Limpopo province.

\section{Material and methods}

A qualitative, phenomenology and exploratory-descriptive design was used to investigate the experience of midwives in implementing HIV guidelines in pregnancy.

\section{Setting}

The study was conducted in the Mopani and Vhembe districts of the Limpopo province (Figure 1) during March to June 2017. The two districts were sampled because of the high MMRs, which were related to the borders or neighboring countries such as Botswana, Zimbabwe, and Mozambique [9]. From the estimated population, about $54.6 \%$ are women, around $45.4 \%$ are men, and $39.4 \%$ are youth. In 1996 , around $42.2 \%$ of population aged 15 years and above were illiterate, with no schooling or highest educational level of less than grade 7 [10]. In 2001, 41.0\% of the population of the same age was illiterate, but a drastic change took place in 2011 , as only $24.0 \%$ of the same population was illiterate, meaning that education was made a priority [10]. There are 223 primary healthcare (PHC) facilities and community healthcare centers within these two districts. Each PHC facility consists of 4 to 7 professional nurs-

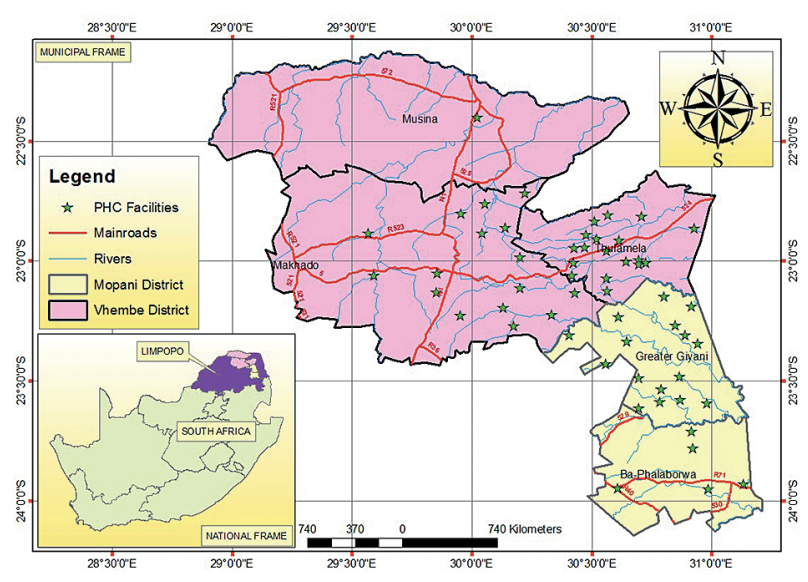

Figure 1. Map of the Limpopo province showing PHC facilities in the Vhembe and Mopani districts 
Table 1. Demographic profile of participants

\begin{tabular}{l|c}
\hline $\begin{array}{l}\text { Variables } \\
\text { Age group (years) }\end{array}$ & $\begin{array}{c}\text { No } \%) \text { of participants } \\
(n=18)\end{array}$ \\
\hline $20-29$ & $1(5.5)$ \\
\hline $30-39$ & $1(5.5)$ \\
\hline $40-49$ & $9(50)$ \\
\hline $50-59$ & $5(27.7)$ \\
\hline$>60$ & $2(11.1)$ \\
\hline Gender & $2(11.1)$ \\
\hline Male & $16(88.8)$ \\
\hline Female & $18(100 \%)$ \\
\hline Position & \\
\hline Professional nurses & $2(11.1)$ \\
\hline No of years in the current position & $2(11.1)$ \\
\hline $2-3$ & $14(77.7)$ \\
\hline $4-5$ & $18(100)$ \\
\hline$>5$ & $18(100)$ \\
\hline Total & $9(50)$ \\
\hline Qualifications & \\
\hline Diploma & $2(11.1)$ \\
\hline Degree & \\
\hline Other & \\
\hline Total & \\
\hline
\end{tabular}

es (PN) and community health centers involve 20-25 PN. The PN's with midwifery qualifications are consulting around 40-45 pregnant women per week, who are coming for prenatal healthcare services. Those who are working at community healthcare centers are registered midwives (5 to 7 ), providing maternal healthcare services, caring for $50-60$ prenatal women, and attending more than 50 deliveries per month. However, PHC facilities are experiencing high-rate of staff shortages, with a huge burden of programs to be implemented [9].

\section{Population and sampling}

Professional nurses with a midwifery qualification, trained on the management of HIV during pregnancy, and who have been working in maternal healthcare services for more than 2 years were included in this study. A heterogeneous purposive sampling method was used, and sampling ensued until data saturation occurred from 18 participants.

\section{Data collection and analysis}

Data was collected from March to June 2017. Appointments for interviews were secured and codes from A to Q were assigned to participants. Data were collected using unstructured, in-depth individual face-to-face interviews, which were conducted in a relaxed conversational manner, with each session lasting for 45-60 minutes. The central question, which guided the interview was: "Can you please share with me your experience of implementing HIV guidelines during pregnancy?". The question was followed by probing as a communication skill, which elicited more information from the participants. Interviews were conducted in English, until no new information was emerging from the $18^{\text {th }}$ participant. Permission to use a voice recorder was obtained, and recordings were transcribed verbatim. Field notes were documented during interviews with given meaning.

Data from unstructured interviews were analyzed qualitatively using Tesch's open coding method [11], starting with the researcher reading carefully through all transcripts to get a sense of all interviews/ information. After the completion of all transcripts, a list of similar topics was collected. Data were grouped according to themes and sub-themes (Table 2), and field notes were also coded and categorized. A literature control was done to contextualize the results of the study [11].

\section{Measures to ensure trustworthiness}

Trustworthiness was ensured through the four principles of Lincoln and Guba's framework, as described by Babbie and Mouton [12]. Credibility was guaranteed by prolonged engagement, which improved the relationship and clarified the descriptions with participants through familiarity. Data triangulation was ensured by using different data collection methods. The researcher collected data through field notes and in-depth individual interviews. Member checking was done to confirm and validate the findings through interviews and discussion with participants in order to evaluate the reality. For dependability, experts were engaged to confirm the methodology, which was further enhanced using an independent coder to ensure consistency. To ensure confirmability, notes were kept safely to enable conduction of an adequate trail and to determine the conclusions, interpretations, and recommendations. Lastly, for transferability, a dense description of methodology was used to ensure that people in other settings and similar projects utilize the same method, and conducted in different environments could generate similar findings.

\section{Ethical considerations}

The ethical clearance to conduct the study was obtained from a research ethics committee of the University of Venda (SHS/16/PBC/34/1910). Limpopo Provincial Department of Health (Ref 4/2/2) as well as District Executive Managers of each district granted permission to access the facilities. Participants gave written, informed consent, and were informed of their right to withdraw from the study without 
Table 2. Themes and sub-themes indicating the experiences of midwives during the implementation of HIV guidelines in pregnancy $(n=18)$

\begin{tabular}{|c|c|}
\hline Themes & Sub-themes \\
\hline $\begin{array}{l}\text { 2.1. Experiences of midwives } \\
\text { during provision of care to } \\
\text { HIV-positive pregnant women }\end{array}$ & $\begin{array}{l}\text { 2.1.1. Difficulty experienced on multiple levels of provision of care to pregnant } \\
\text { HIV-positive women } \\
\text { 2.1.2. Several management procedures employed that lead to delayed care provision } \\
\text { to other women } \\
\text { 2.1.3. Increased workload experienced when carrying for pregnant women who are } \\
\text { HIV-positive }\end{array}$ \\
\hline $\begin{array}{l}\text { 2.2. Management of pregnant } \\
\text { HIV-positive women as per } \\
\text { guidelines }\end{array}$ & $\begin{array}{l}\text { 2.2.1. Treatment guidelines easy to implement to women adhering to ARTs } \\
\text { 2.2.2. Provision of continuous counselling provided to pregnant HIV-positive women }\end{array}$ \\
\hline $\begin{array}{l}\text { 2.3. Challenges experienced by } \\
\text { midwives carrying for pregnant } \\
\text { HIV-positive women }\end{array}$ & $\begin{array}{l}\text { 2.3.1. Patients' complains are related to the length of stay before consultation } \\
\text { in healthcare facility } \\
\text { 2.3.2. Lack of adherence to ARTs leads to difficulty in managing pregnant HIV-positive } \\
\text { women } \\
\text { 2.3.3. Shortage versus availability of consulting rooms }\end{array}$ \\
\hline
\end{tabular}

any penalty. Ethical principles of fairness, privacy, confidentiality, anonymity as well as participants' rights to voluntarily participate in the study were considered.

\section{Results}

Eighteen PNs from PHC facilities of the two selected districts participated in this study (Table 1). Their age ranged between 40 and 49 years, with more than five years working experience as midwives/accoucheurs, which meant that the participants had a vast experience in the management of pregnant women infected with HIV. More than $89 \%$ of the participants were females. Fifty percent of the participants hold of a diploma as their qualification and all were trained in the management of HIV-positive pregnant women. Following the analysis of raw data, subjects that emerged were: experiences of midwives during provision of care to HIV-positive pregnant women, management of pregnant HIV-positive women as per guidelines, and challenges experienced by midwives caring for pregnant HIV-positive women.

\section{Experience of midwives in the provision of care to HIV-positive pregnant women}

Participants reported to perform various procedures during the first antenatal care service; more extensive care is needed during antenatal visits, labor, delivery, and post-natal care if pregnant woman is tested HIV-positive. Participants experienced difficulties, especially with newly diagnosed HIV patients; therefore, they indicated shortage of midwives, which lead to work overloading, especially with one midwife on duty:

"We work being two professional nurses, the other one was not available; hence, I was alone as a midwife with a woman who was coming for her first ANC visit, seeing all patients including minor conditions. I had a challenge of workload. A lay counsellor tested a woman who came for the first visit, the woman was found to be HIV-positive. I had to do post-test counselling again because the woman was difficult to accept her status. But at the end, I was able to prescribe and issue the appropriate drugs including counselling on feeding options and after taking all necessary blood tests" (participant B).

From this theme, sub-themes are indicated in Table 2.

\section{- Difficulty experienced on multiple levels in the provi-} sion of care in HIV-positive women

Participants reported that pregnant women refused referral to home-based care, as other women believed they were bewitched, resulting being HIV-positive. One participant indicated that she came across a pregnant woman who defaulted treatment before her current pregnancy. The woman continued to refuse treatment. As a result, the participant spent more time adhering and counselling the woman, and increased waiting time of other patients:

"The case that I dealt with was a difficult one. It was a P4G6, of 36 years of age, and was a known HIV-positive patient who defaulted treatment for a long time; her last child died a year ago at the age of two years. It was difficult to manage her. It took me hours counselling her and by that time, because we work being two Registered Nurses, the queue was moving slowly, but I am glad I convinced her to take at least the ART drugs" (participant B).

"The one I came across; it was her first visit. The most painful part was that she lived her life well; it was a lady who grew up in the church, got married to a brother 
from the same church. She got married and also got the HIV virus. She came for her first visit and after being tested, her results were reactive. It was a difficult patient, she cried a lot after the results, and it was very tough" (participant F).

"I had a difficult pregnant woman, she was not tested by me, but she went to ZCC church, where they told her that she was bewitched. This was her fifth pregnancy and her third marriage. She believed that she got HIV because she was witched" (participant N).

\section{- Several management procedures employed that lead to delayed care provision of other women}

Participants reported that pregnant women coming for their first antenatal care needs thorough assessment, which included screening of blood for $\mathrm{Hb}, \mathrm{RH}$, and syphilis. In addition, for a pregnant woman who tested positive for HIV, they indicated that baseline bloods are taken in order to assess the legibility of pregnant woman to ART drugs. Participants reported that they counsel, adhere, and dispense ART to pregnant women the same day, and identify those who qualify for initiation of isoniazid preventive therapy:

"I must take baseline bloods, do ART adherence, and then give the woman her drugs. Every positive HIV patient is screened for tuberculosis, so that isoniazid preventive therapy can be given" (participant $C$ ).

"I tested her now and found to be HIV-positive; I evaluated her and if she was not pale, I initiated her on FDC. Then, a baseline blood was taken, including creatinine, Alt, and other blood for ANC" (participant Q).

Some of the participants with the same view of various procedures indicated their experiences as follows:

"I measure vital signs, open ANC card, take ANC blood, $H b, R P R$, and then, counsel and test for HIV. If positive, I staged the patient whether she qualifies for cotrimoxazole. Then initiate her FDC drugs; I also screen for tuberculosis and give ionized drugs, if she does not have TB symptoms" (participant L).

\section{- Increased workload experienced when caring for HIV-} positive pregnant women

Participants reported the implementation of PHC reengineering and IDEAL clinic realization that needs more records to be completed, which consume time. They indicated that it took them one and a half to two hours to manage and complete the records of a pregnant women infected with HIV.

"It takes one hour and 30 minutes to complete all the records. Now with IDEAL clinic, one must complete two books, a new file, and a maternity case register book, and it takes time to complete them" (participant $R$ ).
Another participant agreed with this statement, indicating that she cannot hurry her management, since she needs to give the women all information for better results.

"It takes me an hour to meet and test one woman; I must open a file for her, in which at the end, it takes two hours for me to help her. With other patients waiting, I cannot hurry the patient because I must make sure that I gave her all the appropriate care and information related to her status" (participant $N$ ).

"The problem nowadays is the new file, which takes time to complete; booking the patient and filling her maternity case register is long" (participant Q).

\section{Management of HIV-positive pregnant women as per guidelines}

Participants indicated how they manage pregnant women diagnosed with HIV such as collection of various specimens and screening of opportunistic infection that occur due to a lowered immune system, and this management was seen to be easy in women who either know or suspect that they might be infected. Participants indicated that counselling continues until they are satisfied and sure that the woman have accepted her status.

"I did everything to women who tested HIV-positive, which included collection of ANC blood for $\mathrm{Hb}, \mathrm{Rh}$, and syphilis as well as baseline blood for HIV. I screened for $T B$, so that I can issue INH. It is easy if a woman suspects herself of being infected, but for a woman who was negative previously and now tested HIV-positive, it is difficult to accept her present status. I continue to counsel her until we reach an agreement. That is when I issue ART drugs and give her follow-up dates" (participant $O)$.

Below are sub-themes that emerged from this theme.

\section{- Treatment guidelines easy to implement to women ad- hering to ART}

Participants indicated the simplicity of implementing the guidelines in pregnant women who knew their status before they fell pregnant; hence, these women took their ART drugs without a problem. Pregnant women who discovered that they were HIV-positive on their first ANC visit experienced difficulty, therefore it was difficult to manage such pregnant women. From the past experience of the researcher, women who were diagnosed a long time ago are free to talk about their HIV status in public and are employed as mother mentors at PHC facilities.

"The woman that I found being HIV-positive and knows her status will not give you a problem because she got pregnant being aware of her status than a woman who found herself HIV-positive at that time. Women 
who get pregnant knowing their status does not take much time, but for a newly diagnosed woman, the visit take a long time" (participant K).

"It becomes simple to implement the guidelines, if one has started by attending her ANC booking first, lastly do PMTCT, though completion of ANC booklet becomes difficult with the patient not accepting her condition" (participant $C$ ).

Another participant indicated her success in implementing the guidelines:

"Nowadays people understand the program. Even if you start counselling the patient about HIV and they are 10 patients, all of them will be tested. No one will decline and when one needs to initiate the drugs, they all will accept" (participant $\mathrm{O}$ ).

\section{- Provision of continuous counselling provided to HIV- positive women}

Participants reported that they offer continuous counselling to those diagnosed with HIV in order to monitor the psychological state of pregnant women. The importance of follow-up visits was indicated where midwives check the adherence to ART drugs and any side effects experienced:

"That is why after being seen, the woman must be given a return day, which is soon, in order to assess her condition if she is still at the same denial state or not" (participant $K)$.

One participant agreed and further indicated that she offered reassurance that in time, side effects will subside as a means of encouraging women to continue taking ART drugs:

"When women come back for follow-up, I ask them about their experiences in relation to treatment and side effects, and some will say they did not experience any, though some will tell you that I had mild diarrhea. I reassured them that it will pass, but it is difficult especially for a first time diagnosed patient" (participant J).

Another participant indicated an issue of checking the blood results in order to see whether a woman can continue with the prescribed drugs or not:

"The patient is supposed to come back within 7 days for the results to check for creatinine, in particular. It can be down from 30-35 and it does not allow me to give drugs. A woman qualifies to get three drugs; if her creatinine starts from 40, though it will still be low, it allows me to give drugs" (participant A).

\section{Challenges experienced by midwives/accoucheurs caring for HIV-positive women}

All participants reported challenges such as long waiting periods, when providing maternal healthcare services to pregnant women diagnosed with HIV. This was influenced by many procedures that need to be performed during the visit:

"The waiting period is long, sometimes it takes 1 hour especially for the one who tested positive. I have to counsel the woman for adherence of drugs, issue the drugs, take all necessary blood for testing creatinine, Alt, and viral load" (participant K).

\section{- Patient complaints are related to length of stay before consultation in healthcare facility}

It was reported that other patients complained about the waiting period, which delayed them from receiving PHC services. A delay was influenced by shortage of staff, where one midwife/accoucheur must see all patients who came for consultations. Some participants indicated that they apologize to patients and explained the reason for the delay:

"Some patients do complain, but I explain the reason for the situation, since I must help this woman in all matters. Patients do complain, but I communicate with them" (participant $P$ ).

"It takes a lot of time, if you are working alone that day seeing pregnant women and minor ailments. I have to go out and apologize to patients waiting outside, because patients outside can have other thoughts on what is happening inside. It is better if other midwives like three are on duty rather than being alone" (participant $L$ ).

\section{- Lack of adherence to ART leads to difficulty in manag- ing HIV-positive women}

The National Consolidated Guidelines on PMTCT of 2015 stated that disclosure of one's status promote adherence to drugs, hence participants indicated that pregnant women failed to disclose their status to their husbands and as such failed to take their drugs. Participants reported that some women, because of their religion, believed in witchcraft as told by their pastors, and denied their results and drug taking.

"I have come across this woman where I worked previously. The patient was attended by another nurse, after being tested positive, the women refused ARV treatment and said she is a member of a Zion Christian church and she will go to the church. Moreover, her pastor already has seen her condition, and it was not true" (participant K).

"They have a problem on how to take the drugs, where to store them, whether inside a bag of maize meals, so it is a challenge because we stress that ARVs must be tak- 
en at the same time every day without skipping. Their problem is the disclosure of their condition to their husbands, so they do not take the drugs" (participant N).

Participants indicated that another pregnant woman was convinced by family members to use traditional drugs instead of ARV. Therefore, midwives had to conduct home visits to counsel the members in order to enroll the patient in an ARV program:

\begin{abstract}
"After the delivery, at home, the in laws advised the woman to leave ART drugs and use traditional medicines. She did that for one week, then she came back to the facility and she was fortunate I was there. She told me her story, and we had to do home visit again. We found the woman, husband, and mother in law at home. We did re-counselling of all as a family and explained the importance of taking drugs. From there, she started to take her drugs again with no problems" (participant F).
\end{abstract}

\section{- Shortage versus availability of consulting rooms}

Some of the participants reported and complained about infrastructure due to shortage of consulting rooms. Some of the facilities, having three midwives, were also experiencing the same problem, because they had only two consulting rooms, and IDEAL clinic realization required three consulting rooms for the facility to offer ideal health care services. follows:

Participants encapsulated the issue of infrastructure as

"Our infrastructure is small; all in all, we are six professional nurses, most of the time three professional nurses are on duty and cubicles are small, as we are having three streams. Something can be done to increase our infrastructure, so that we have enough space to work" (participant $\mathrm{O})$.

Another participant agreed and indicated that:

"Improved infrastructure, because the one we are having does not accommodate all the staff in our facility; there are a lot of services and diseases; we need to adjust lay counsellors, HIV mentors; our infrastructure cannot accommodate all those services" (participant L).

Another participant who agreed, added the issue of provision of staff in order to improve healthcare services:

"Infrastructure is the problem; if our infrastructure can be increased and enough staff provided, it would make our and patients' lives improved" (participant D).

\section{Discussion}

The findings of this study revealed that midwives experienced difficulty in the management of pregnant women who were coming for antenatal appointments for the first time and tested positive for HIV. As a normal reaction, it was expected that pregnant women reject positive results when one had a negative status for a long time. Various authors and the Centers for Disease Control and Prevention (CDC) indicated that midwives must expect that the patient will be distressed and difficult to handle when she is informed of the positive result [13-15]. Researchers indicated that the patients need to adjust to new changes in their lives, learn how to cope with the virus, how to tell their families, and how to handle the fear of stigma. They further indicated that the patients need some help on how to adapt to all the aforementioned changes. Sometimes women can be affected psychologically and hence, need to be referred to a psychologist [13-16]. The implication of the difficulties experienced could lead to treatment failure, because if a woman who does not believe that she is HIV-positive, would not take drugs for the treatment of the virus. Therefore, the virus will continue to replicate and lower the immune system, especially in pregnant women who are prone to opportunist infections and increased MMR.

There were several management procedures performed in pregnant women coming for their first antenatal care appointment, which included blood screening for $\mathrm{Hb}, \mathrm{Rh}$, and syphilis. In addition, in a pregnant woman who was tested HIV-positive, the baseline blood is additionally taken in order to assess her suitability to ART drugs [17]. Furthermore, the PMTCT guidelines suggested that screening of tuberculosis must be done to identify women who qualify for initiation of isoniazid preventive therapy [8]. Implementation of several procedures in the PMTCT by midwives lead to delayed care of other types of patients like those with minor or chronic ailments; although its benefits will result in suppressed viral load and the delivery of a virus-free baby. In case of shortage of staff, several procedures could cause burnout syndrome and work overload, and not all procedures could be performed correctly, resulting in poor implementation of the guidelines. It was discovered that several procedures performed on HIV-positive pregnant women predisposed midwives to infections caused by work overload and shortage of staff [18]. A study, which was conducted in Zimbabwe amongst healthcare workers on the implementation of HIV guidelines in pregnancy indicated that several programs lead to poor implementation of the guidelines, resulting in poor performance $[19,20]$. Different records were completed for a pregnant woman who tested positive for HIV, which led to an increased workload. Although, the department of health tried to reduce the workload of midwives by hiring data captures and lay counsellors, there is a need to strengthen the workforce that will focus on HIV only. Work overload results in stress, which leads to poor implementation of the guidelines. Findings of various studies revealed stress amongst midwives caused by work overload and therefore, poor maternal outcomes [21,22].

The Maternity Care guidelines used in South Africa and PMTCT guidelines indicate that a pregnant woman diagnosed with HIV must have blood taken, receive ART coun- 
selling adherence, and be screened for opportunistic infection such as TB; if TB is detected, TB drugs to be given and if no signs found, an isoniazid preventive therapy (IPT) to be initiated [2]. The findings showed that midwives were following guidelines when managing pregnant women with HIV, as they indicated baseline blood collections and issuing of ARV drugs on the same day. However, they were facing challenges such as shortage of staff. Further findings indicated that treatment guidelines were easy to implement in HIV pregnant women, who were adhering to ART treatment, similar to the CDC that revealed simplicity of guideline implementation by healthcare providers. Most patients who were on ARVs had their viral load suppressed because of adherence to treatment [23]. Various researchers identified factors that can influence success or failure of patients' adherence to ART: the relationship between the healthcare providers and the patient, the patient's socioeconomic status, health condition, and the types of drugs that the patient is taking [23-25]. The implications of treatment guidelines implementation by midwives to women who were adhering to ART would improve maternal health status, hence promoting a healthy community. As indicated by the National Consolidated Guidelines on PMTCT (2015), findings revealed that continuous counselling of women diagnosed with HIV was done within seven days after the initial diagnosis. This was completed in order to assess if pregnant women have accepted their status and to check for side effects of ART drugs. Several authors indicated that continuous counselling of women who had been diagnosed with HIV helps to evaluate if women accepted their conditions and how they cope with it. It also helps to keep track of treatment compliance [26, 27]. The emotional status of women was also assessed to determine if there was a need for referral for further counselling. Women were taught strategies to reduce the risk of HIV transmission to reduce MMR $[10,28]$. The implications of continuous counselling in pregnant women living with HIV would help to understand their condition, while improving adherence to ART.

Midwives and accoucheurs were faced with challenges when implementing PMTCT guidelines. A cited challenge was patients complained about the long waiting period in the facility before consultation with midwives. Long queues at PHC facilities were caused by a shortage of human resources, as indicated by several authors. This leaded to a delay in initiating the treatment, therefore patients were dissatisfied [28-30]. Similar results were discovered in a study conducted on patients in a rural region in South Africa, where patients were given appointment dates for consultation to avoid long waiting periods [29-32].

Lack of adherence to ART drugs by pregnant women was another challenge experienced by midwives, thus it was difficult for them to manage HIV-positive pregnant women. Financial constraints, stigma associated with HIV, problems in a health institution, and poor adherence to drugs were identified as barriers that lead to poor adherence to the treatment [27]. Staff attitudes, physical abuse, lack of privacy, poor communication, and patients ignoring were other causes of poor adherence to drugs by pregnant women [33].
The implications of lack of adherence would lead to an increased viral load and lowered immune system, followed by increased MMR. As identified in our study, shortage of consulting rooms was similar to that of study conducted in Ghana. This led to a delay in the provision of maternal healthcare services [18]. Shortages of consulting rooms, as identified by various authors, leads to an increase in patient waiting times $[18,34,35]$. The implication of the shortage of consulting rooms would influence pregnant women to seek alternative ways of healing, such as consulting traditional healers, so their viral load would rise.

\section{Limitation of the study}

Only two districts were involved in this study. Findings cannot be generalized to other three districts of the Limpopo province.

\section{Recommendations}

The strengthening of counselling skills of midwives as a strategy to facilitate adherence to ARV by pregnant women is recommended. The involvement of family members and community during continuous education and training are also recommended, so that HIV/AIDS is perceive as a chronic condition to be treated, not stigmatized. The recommendations should be shared with the District Manager to strengthen the quality and quantity of the workforce. Hiring of more staff will provide HIV programs and reduce patient waiting times. Consolidation of registers and provision of electronic gadgets are also recommended to reduce the use of different registers for filing patients' records. Facilitating of the procurement of consulting rooms (park homes) and revitalization of infrastructure to accommodate three streams are recommended in order to reduce waiting times. Researchers further recommended revision of policies of referral and transfer logistics.

\section{Conclusions}

Midwives are implementing the HIV guidelines during pregnancy; however, shortage of human resources during the first visit results in a prolonged waiting time for other patients. They are also expected to complete different records, which takes more time to consult one patient when there are fewer midwives. This is further perceived as increased workload. Midwives are experiencing challenges related to prolonged turnaround time for the patients' results and shortage of consulting rooms. This affects the clinical decision of care. Non-adherence amongst patients affected the provision of HIV care.

\section{Acknowledgements}

Authors would like to acknowledge all midwives from PHC facilities who participated in this study as well as the South African Medical Research Council and University of Venda for funding this study. 


\section{Conflict of interest}

The authors declare no conflict of interest with respect to the research, authorship, and/or publication of this article.

\section{References}

1. Kasenga F, Byass P, Emmelin M, Hurtig AK. The implications of policy changes on the uptake of a PMTCT programme in rural Malawi: first three years of experience. Glob Health Action 2009; 2. doi: 10.3402/gha.v2i0.1883.

2. NDoH. National Consolidated Guidelines for the prevention of Mother to Child Transmission of HIV (PMTCT) and the Management of HIV in children, Adolescents and Adults, South Africa 2015.

3. DoH Statistics. Tenth Interim Report on confidential enquiries into maternal deaths in South Africa, Pretoria. Government Printers 2014

4. Mnyani CN, McIntyre JA. Challenges to delivering quality care in a prevention of mother-to-child transmission of HIV programme in Soweto. Southern African Journal of HIV Medicine 2013; 14: 64-69.

5. WHO. Trends in maternal mortality rate from 1990-2013. Estimates by WHO, UNICEF, UNFPA, The World Bank and the United Nations Population Division. 2014.

6. NDoH. National Consolidated Guidelines for the Prevention of Mother-to-child Transmission of HIV (PMTCT) and the Management of HIV in Children, Adolescents and Adults. South Africa 2014.

7. DoH. Saving Mothers 2011-2013: Sixth Report on Confidential Enquiries into Maternal Deaths in South Africa, NCCEMD, Pretoria. Government Printers 2014.

8. DoH. Saving Mothers 2014-2016: Seventh Report on Confidential Enquiries into Maternal Deaths in South Africa, NCCEMD, Pretoria. Government Printers 2017.

9. Massyn N, English R, McCracken, et al. Disease profile for Vhembe Health District, Limpopo. Durban: Health Systems Trust. 2015.

10. Census, 2011. Statistical release. Available at: http://www.statssa. gov.za/publications/P03014/P030142011. Accessed on 20.02.2019

11. Creswell JW. Research Design Qualitative, Quantitative, and Mixed Methods Approaches. SAGE Publications, Inc. 2018.

12. Babbie E, Mouton J. The Practice of Social Research. $10^{\text {th }} \mathrm{ed}$. Republic of South Africa, Oxford University Press Southern Africa, Cape Town 2010.

13. Braddick L, Tuckey V, Abbas Z, et al. A mixed-methods study of barriers and facilitators to the implementation of postpartum haemorrhage guidelines in Uganda. Int J Gynaecol Obstet 2016; 132: 89-93.

14. CDC. Monitoring selected national HIV prevention and care objectives by using HIV surveillance data - United States and 6 US dependent areas - 2010. 2012

15. Warner L, Klausner JD, Rietmeijer CA. Effect of a brief video intervention on incident infection among patients attending sexually transmitted disease clinics. PLoS Med 2008; 5: e135.

16. CDC. Monitoring selected national HIV prevention and care objectives by using HIV surveillance data - United States. 2017.

17. Koss CA, Dunne EF, Warner L. A systematic review of epidemiologic studies assessing condom use and risk of syphilis. Sex Transm Dis 2009; 36: 401-405.

18. O'Connor A, Doris F, Skirton H. Midwifery care in the UK for older mothers. Br J Midwif 2014; 22: 568-577.

19. Banchani E, Tenkorang EY. Implementation challenges of maternal health care in Ghana: the case of health care providers in the Tamale Metropolis. BMC Health Serv Res 2014; 14: 7.

20. Ngxongo TS, Sibiya MN. Factors influencing successful implementation of the basic antenatal care approach in primary health care facilities in eThekwini district, KwaZulu-Natal. Curationis 2013; 36 : E1-7.

21. Mathole T, Labonté R, Sanders D, et al. Health worker migration from South Africa: causes, consequences and policy responses. Hum Resour Health 2015; 13: 92.
22. Scheffler E, Visagie S, Schneider M. The impact of health service variables on health care access in a low resourced urban setting in the Western Cape, South Africa. Afr J Prim Health Care Fam Med 2015; 7: 820 .

23. Tibandebage P, Kida T, Mackintosh M, Ikingura J. Can managers empower nurse-midwives to improve maternal health care? A comparison of two resource-poor hospitals in Tanzania. Int J Health Plann Manage 2016; 31: 379-395.

24. Thompson MA, Mugavero MJ, Amico KR, et al. Guidelines for improving entry into and retention in care and antiretroviral adherence for persons with HIV: evidence-based recommendations from an International Association of Physicians in AIDS Care panel. Ann Intern Med 2012; 156: 817-833.

25. Cohen MS, Chen YQ, McCauley M, et al. Prevention of HIV-1 infection with early antiretroviral therapy. N Engl J Med 2011; 365: 493505.

26. Metsch LR, Feaster DJ, Gooden L, et al. Effect of risk-reduction counseling with rapid HIV testing on risk of acquiring sexually transmitted infections: the AWARE randomized clinical trial. JAMA 2013; 310: 1701-1710.

27. Aberg JA, Gallant JE, Ghanem KG, et al. Primary care guidelines for the management of persons infected with HIV, update by the HIV Medicine Association of the Infectious Diseases Society of America. Clin Infect Dis 2013; 58: e1-e34.

28. Baloyi LF. Problems in providing primary health care services: Limpopo Province. Doctoral dissertation, University of South Africa. 2010.

29. Daniels JA. Assessing the impact of a waiting time survey on reducing waiting times in primary care clinics in Cape Town, South Africa. Doctoral dissertation, University of Cape Town. 2015.

30. Moses KS. The perceptions of clinical nurse practitioners on service delivery in primary health care facilities in Eden District, Western Cape. Doctoral dissertation, Stellenbosch University, Stellenbosch 2017.

31. Saburi S. Experiences and perceptions of Zimbabwean migrant women accessing antenatal and infant/child immunisation in public health care services in Gauteng South Africa (2015-2017). Doctoral dissertation. 2017.

32. Visagie S, Schneider M. Implementation of the principles of primary health care in rural area of South Africa. Afr J Prim Health Care Fam Med 2014; 6. doi: http://dx.doi.org/10.4102/phcfm.v6i1.562.

33. Mahnava P, Durrant K, Luchters S. Attitude and behaviours of maternal health care providers in interactions with clients: a systemic review. NCBI-NIH 2015. Available at: https://www.ncbi.n/m.nih.gov.

34. Cameron D, Gerber A, Mbatha M, et al. Nurse initiation and maintenance of patients on antiretroviral therapy: are nurses in primary care clinics initiating ART after attending NIMART training? S Afr Med J 2012; 102: 98-100.

35. Pearce C, Hall S, Phillips C, et al. A spatial analysis of the expanding roles of nurses in general practice. BMC Nurs 2012; 11: 13. 